Division of Research

February 1981

Graduate School of Business Administration

The University of Michigan

SELECTION PROCEDURES: A NEW STATISTICAL

METHODOLOGY AND ITS APPLICATIONS FOR

MARKETING RESEARCH

Working Paper No. 251

Jean Dickinson Gibbons

The University of Alabama

Oded Gur-Arie

The University of Michigan

FOR DISCUSSION PURPOSES ONLY

None of this material is to be quoted or reproduced without the express permission of the Division of Research. 


\title{
SELECTION PROCEDURES: A NEW STATISTICAL METHODOLOGY AND ITS APPLICATIONS FOR MARKETING RESEARCH
}

\author{
Abstract \\ Traditional methods of analysis do not provide the \\ researcher with means for selecting the "best" among \\ several alternatives while having control over the \\ probability of being correct. This paper presents the \\ indifference zone approach and the subset-selection approach \\ to selection problems. The findings of several recent \\ articles are used as illustrative examples.
}




\section{SELECTION PROCEDURES: A NEW STATISTICAL \\ METHODOLOGY AND ITS APPLICATIONS \\ FOR MARKETING RESEARCH}

\section{Introduction}

Researchers in marketing often have to select, based on some well defined criteria, among several possible alternatives. They may have to decide on issues such as which advertising theme is most effective, which of several product designs is most preferred by consumers, which of several geographic regions has the highest sales potential, or which questionnaire design will yield the highest response rate. In such cases, the researcher's basic objective should be to determine which of the several alternatives is best in some well defined sense, along with an appropriate estimate of the probability that the selection is correct.

The traditional research approach, however, centers on testing a null hypothesis of no difference among the several populations with respect to some parameter. If that hypothesis is rejected, it is usually concluded that the alternatives are not all equivalent. The research objective of selecting the best alternative, with control over the probability of being correct, is not met. 
The purpose of this paper is to present the methodologies for the statistical selection of populations, as well as illustrative examples. The methodology for selecting populations is aimed at selecting among several populations the one which is best for some desired result, while having control over the probability of making a correct selection. Furthermore, whenever the research objective is to evaluate and'select among different alternatives, it is reasonable to expect that no matter how slight the differences among those alternatives are, a sufficiently large sample size will establish those differences. The selection procedures described here provide the means for determining the minimum number of observations needed to establish that the probability of a correct selection is at least some specified value. The theory of selecting populations was originated in the 1940s "by Wald's work on sequential analysis (1947) and subsequent work by Girshick (1946). Procedures for the selection of the best population were first presented by Bechhofer (1954) and Bechhofer, Dunnett, and Sobel (1954). Procedures for selecting a subset of populations that contains the best population were developed by Gupta (1956). Since then the procedures have been developed for many goals and many distributions; the work in this area, however, has been mainly in the theoretical literature and, hence, unavailable to researchers in practical form until Gibbons, Olkin, and Sobel (1977). 


\section{The Concept of Selection}

Suppose there are $\mathrm{k}$ populations indexed by a parameter $\theta$ as

$$
f\left(x_{1} ; \theta_{1}\right), f\left(x_{2} ; \theta_{2}\right), \ldots, f\left(x_{k} ; \theta_{k}\right)
$$

where $\theta_{j}$ is the parameter of the $j$ th population. It is assumed that the populations do not all have the same $\theta$ values and that they can be ordered in some meaningful way with respect to the values of $\theta$. It is further assumed that the "best" population is the one with the largest $\theta$ value and therefore the research problem is to identify that population among the $\mathrm{k}$ populations.

Denoting the ordered values of $\theta$ as

$$
\theta_{[1]} \leq \theta_{[2]} \leq \cdots \leq \theta_{[k]}
$$

we wish to identify the value of $j$ among $j=1,2 \ldots k$, for which the parameter value of $\theta_{j}$ is equal to $\theta_{[\mathrm{k}]}$. The selection procedure for such a problem is to take a random sample from each of the $k$ populations, compute $\hat{\theta}_{j}$, an estimator of $\theta_{j}$, for each sample, and assert that the "best" population is the one for which the value of $\hat{\theta}$ is largest.

At issue here is not the estimate of $\theta_{[\mathrm{k}]}$, but rather the selection or the identification of that population with $\theta$ value equal to $\theta_{[\mathrm{k}]}$. Therefore, the only possible error occurs if the selection is incorrect. Whenever $\theta_{[k]}>\theta_{[k-1]}$ ' there is only one selection that is correct. The probability of making the correct selection can be seen as the analog of 
the $1-\beta$ probability (power of the test) in classical hypothesis testing. A good selection procedure therefore is one for which the probability of a correct decision is large (say, .9 and above). This probability in turn can be interpreted as the confidence we have in the selection procedure.

The probability of a correct selection (CS) under an arbitrary vector or configuration $\theta$ can be denoted as $\mathrm{P}\{\mathrm{CS} \mid \theta\}$. If there are two different selection rules, say $\mathrm{R}_{1}$ and $\mathrm{R}_{2}$ with corresponding $\mathrm{P}\{\mathrm{CS} \mid \theta\}$ functions $\mathrm{P}_{1}$ and $\mathrm{P}_{2}$, it is likely that $\mathrm{P}_{1} \geq \mathrm{P}_{2}$ for some sets of $\theta$ values and $\mathrm{P}_{1} \leq \mathrm{P}_{2}$ for others. Therefore, in order to set up criteria for a good selection rule; the entire space of the parameter values should be analyzed, and those regions where the researcher has a strong preference for making a correct selection should be separated from those regions where the researcher is indifferent between the two or more selections. The former region is referred to as the preference zone (PZ), while the latter is referred to as the indifference zone (IZ).

Suppose there are $k=2$ populations and $\theta_{1}$ and $\theta_{2}$ can be any real numbers so that the entire two-dimensional plane is the parameter space. If some specified $\delta *>0$ is the smallest difference between $\theta_{1}$ and $\theta_{2}$ that is worth bothering about, then $\left|\theta_{2}-\theta_{1}\right| \geq \delta^{*}$ is the preference zone while $\left|\theta_{2}-\theta_{1}\right|<\delta *$ is the indifference zone, the region where the values of $\theta_{1}$ and $\theta_{2}$ are so close that the researcher may be indifferent about which one is larger. These zones might appear as in Figure 1. 
Figure 1 about here

For any arbitrary $\mathrm{k}$ the entire parameter space is a region with $\mathrm{k}$ dimensions. However, the selection of $\theta_{[\mathrm{k}]}$ is the main concern; therefore, the researcher should be concerned with how much $\theta_{[\mathrm{k}]}$ is larger than $\theta_{[\mathrm{k}-1]}$ and on $l_{y}$ to a lesser degree with their values relative to $\theta_{[1]}, \ldots ., \theta_{[\mathrm{k}-2]} \cdot \mathrm{H}$ Hence, the definition of the $\mathrm{PZ}$ and IZ need depend only on $\theta_{[k]}$ and $\theta_{[k-1]}$ and the zones can be depicted by a two-dimensional diagram as in Figure 1, no matter what is the value of $\mathrm{k}$.

In order to measure the distance between the two parameters, there is a need to define some function $\delta$ of $\theta_{[\mathrm{k}]}$ and $\theta_{[\mathrm{k}-1]}$ as well as a threshhold value $\delta *$ to separate the IZ from the $\mathrm{PZ}$. The function $\delta$ need not be the difference of $\theta_{[k]}$ and $\theta_{[k-1]}$. For instance, if the distributions are binomial, $\delta$ might be defined as the so-called odds ratio-the odds for success in the best population divided by the odds for success in the next best. Then the parameter values are limited to the closed interval $[0,1]$ and the preference zone is shown in Figure 2 as:

$$
\theta_{[k]}\left(1-\theta_{[k-1]}\right) /\left(1-\theta_{[k]}\right) \theta_{[k-1]}{ }^{>} \delta^{*}
$$


Figure 2 about here

Generally, there may be some special configuration in the preference zone for which the probability of a correct decision is a minimum over all $\theta$ in the PZ. This configuration is called the least favorable configuration (LF) denoted by

$$
\theta_{\mathrm{LF}}=\left(\theta_{1, \mathrm{LF}}, \theta_{2, \mathrm{LF}}, \cdots, \theta_{\mathrm{k}, \mathrm{LF}}\right)
$$

The probability of a correct selection for any configuration in the preference zone is then at least as large as the probability of a correct selection for the LF configuration, or in symbols:

$$
\mathrm{P}\{\mathrm{CS} \mid \theta\} \geq \mathrm{P}\left\{\mathrm{CS} \mid \theta_{\mathrm{LF}}\right\} \text { for all } \theta \in \mathrm{PZ} \text {. }
$$

If $\mathrm{P}\left\{\mathrm{CS} \mid \theta_{\mathrm{LF}}\right\}$ is some specified value, say $\mathrm{P}$, then the probability of a correct selection $P\{C S \mid \theta\}$ is at least $P^{*}$ for any $\theta$ in the $\mathrm{Pz}$.

In general, $\delta^{*}$ and $P^{*}$ are preset by the researcher (e.g., $\delta^{*}=.3$ and $\left.P^{*}=.95\right)$. In order to satisfy the requirement

$$
\mathrm{P}\{\mathrm{CS} \mid \theta\} \geq \mathrm{P} * \text { for all } \delta>\delta *, \backslash
$$

there is a need to also determine the required common sample size $\mathrm{n}$ per population. Generally, the value of $\mathrm{n}$ is determined by using the equality

$$
\mathrm{P}\left\{\mathrm{CS} \mid \theta_{\mathrm{LF}}\right\}=\mathrm{P}^{*} \text {. }
$$


Tables that relate the values of $n, \delta *, P^{*}$ are available (Gibbons, Olkins, and Sobel 1977) for most common distri-. butions such as the normal, binomial, multinomial and so on, as well as for some nonparametric problems. For example, Tables 1 and 2 of this paper apply to the problem of selecting the one of $\mathrm{k}$ binomial populations with the largest probability of success for $\mathrm{k}=3$ and $\mathrm{k}=4$, respectively. The table entries are the values of $\mathrm{n}$ required under the least favorable configuration; here the preference zone is defined by $p_{[k]}-p_{[k-1]} \geq \delta *$ where $p_{i}$ is the true probability of a success in the ith binomial population. Similar tables are available for the multinomial distribution with $\mathrm{k}$ possible outcomes where the goal is to select the outcome with the largest probability of success. For the normal distribution, the tables usually do not give $\mathrm{n}$ directly. Table 3 is a good example. It applies to the problem of selecting the one of $\mathrm{k}$ normal populations with the largest mean where the normal populations all have the same, known variance. The appropriate entry in Table 3, labeled $\tau$, is substituted in the formula

$$
\mathrm{n}=\sigma^{2}(\tau / \delta *)^{2}
$$

to give the sample size required under the least favorable configuration where $\delta *$ is given by the specified preference zone $\mu_{[k]^{-\mu}}[k-1]^{\geq \delta^{*}}$. The result here generally is not an integer; a Iecimal value should be rounded up to the next larger integer to ensure a conservative sample size. 
For all of these problems, once $\mathrm{n}$ is determined, a confidence statement can be made about the selection. This statement is that with confidence level $\mathrm{P}^{*}$, the $\theta$ value of the population selected, $\theta_{S}$, satisfies the inequality

$$
\theta_{[k]}-\delta * \leq \theta_{S} \leq \theta_{[k]}
$$

This says that the parameter value of the population selected is at most $\delta *$ smaller than the largest parameter value.

Alternatively, for a given sample size, the researcher can use the available tables to calculate the operating characteristic curve of the selection procedure that applies. This curve is the locus of $\left(\delta^{*}, \mathrm{P}^{*}\right)$ values that are satisfied by the selection procedure for that sample size.

Thəse procedures are eminently appropriate for the solution of many problems in marketing research. They enable the researcher to know the minimum probability of a correct selection for a given sample size, and tell how that probability varies as the sample size changes. Furthermore, in designing experiments these procedures provide a rational basis for the determination of the sample size required to determine that the probability of a correct selection is at least some specified value.

In order to illustrate the applications of selection procedures, several recent articles are reviewed. The purpose here is not to criticize the articles, but rather to show the need for familiarity with the techniques. 
Examples of Application

Robertson and Bellenger (1978) studied the relative effectiveness of two types of promised incentives in eliciting response from a mail survey. These incentives were:

1. Personal Incentive - $\$ 1.00$ cash payment

2. Charity Incentive - \$1.00 contribution to their chosen charity.

To carry out the study, the authors divided a systematic sample of 450 Denver area residents into three groups of 150 each. All persons received identical questionnaires. Each member of the first group was offered no incentive for response; the second group was promised the Personal Incentive; the third group was promised the Charity Incentive. The response rates of the three groups were:

$$
\begin{array}{ll}
\text { No Incentive } & 23.3 \% \\
\text { Personal Incentive } & 26.0 \\
\text { Charity Incentive } & 41.3
\end{array}
$$

The authors used these data to perform three chi-square tests by pairs and concluded from the results that the response rate of the Charity Incentive group was significantly higher than that of either the Personal Incentive or the No Incentive groups. This conclusion implies that the appropriate goal in this study was to select the one group with the highest response rate. A chi-square test that rejects the null hypothesis of homogeneity cannot, however, justify such a selection because that test always applies to a two-sided alternative and hence permits only the conclusion that the groups are not 
the same. Further, the performance of three chi-square tests on a single set of data increases the overall significance level of the procedure and a chi-square test is always approximate. The methods presented in this paper are the only ones that can accomplish the implied goal and justify the stated conclusion and they are exact methods.

Suppose first that we are designing an experiment to select the group with the highest response rate here where there are $k=3$ groups. Suppose further that we specify that we want the probability that our selection is correct to be at least .95 when the response rate of the best group is at least $5 \%$ more than the response rate of the other two groups. Thus we have specified $\mathrm{P}^{*}=.95$ and $\delta *=.05$ for $\mathrm{k}=3$. Table 1 shows that $\mathrm{n}=735$ observations are needed for each group in order to have this strong assurance that our selection will be correct. If this solution seems impractical, we might decide to be satisfied with the specification $\mathrm{P}^{*}=.90$ and $\delta *=.10$. Table 1 shows that in this case only $\mathrm{n}=125$ observations are required for each group.

On the other hand, suppose the data are already obtained as indicated in the article and $n=150$ observations are taken for each group. Then we can interpolate in Table 1 with $\mathrm{n}=150$ to find the $\left(\delta^{*}, \mathrm{P}^{*}\right)$ pairs that are satisfied in this experiment. Figure 3 shows these pairs as the operating characteristic curve of the selection procedure for this problem. For example, if the true response rate of the 
best group differs from that of the next best group by at least $14.7 \%$, the probability is at least .99 that the selection is correct. Therefore, since in the reported study the difference between the Charity Incentive group and the Personal Incentive is $15.3 \%$, the authors' conclusion is reinforced. However, while their conclusion was reached via an indirect approximate method, the selection procedure illustrated here provides us with the exact ninimum probability of a correct selection!

Figure 3 about here

A similar study of mail survey response rates was carried out by Goodstadt, Chung, Kronitz, and cook (1977). A random sample of 2,416 magazine readers were to be sent an advance letter concerning a forthcoming questionnaire concerning the magazine. "The sample was then divided into the following four incentive groups of size 604 each to receive the questionnaire:

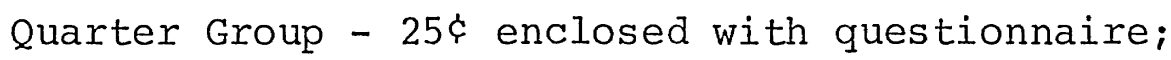

Free Book Group - free copy of book enclosed with questionnaire;

Book Reward Group - free copy of book promised after receipt of completed questionnaire;

Control Group - no incentive.

The authors performed a chi-square test of all the response rates as well as individual comparisons between pairs of groups and concluded that the Quarter Group showed a 
significantly larger response rate than any of the others. Such a directional conclusion can be more properly justified statistically by using the selection procedures of this paper. If the goal in the experiment is to select the one of $k=4$ groups with the largest response rate, the $n=604$ sample size per group will permit high confidence that the group selected is truly the best group. For example, Table 2 shows that with confidence .95 , the response rate of the Quarter Group is within approximately .06 of the response rate of the best group.

Whenever the research objective is to evaluate and select among different alternatives, it is reasonable to expect that no matter how slight the differences among the alternatives are, a sufficiently large sample size will establish those differences. The procedures presented here provide the means for determining the minimum number of observations needed to establish that the probability of a correct selection is at least some specified value for parameter configurations in the preference zone. For instance, if the researchers in the previous example would have been satisfied to establish with confidence .95 that the response rate of the Quarter Group is within .10 of the response rate of the best group then a sample size of only 212 per group would have been sufficient.

Frequently in practice, the groups may not all be of the same size. Even if the group sizes are all the same when the mail survey is initiated, some questionnaires may be undeliverable or returned unopened so that the actual 
sample sizes are unequal. If the sample sizes $n_{1}, n_{2}, \ldots$, $\mathrm{n}_{\mathrm{k}}$ for the $\mathrm{k}$ groups are unequal but are not too disparate in magnitude, the exact same procedure gives approximate solutions for $\left(\delta^{*}, \mathrm{P}^{*}\right)$ with the square-mean-root sample size computed from

$$
\bar{n}=\left[\left(\sqrt{n}_{1}+\sqrt{n}_{2}+\ldots+\sqrt{n}_{k}\right) / k\right]^{2}
$$

Examples of other studies for which these procedures would have been more appropriate than chi-square or t-tests are Goldstein and Friedman (1975), Lipstein (1975), Shosteck (1975), Orpen (1975), and Goulet (1977).

\section{Subset Selection}

Sometimes the purpose of a marketing research study of response rates is only to select those forms of requests or incentives that produce a response rate better than that of a control group. This type of goal is called a subset selection problem and its solution has also been worked out in the statistical literature. In this case, there is no indifference zone and no sample size solutions are given for specified $\left(\delta *, \mathrm{P}^{*}\right)$ values. Rather, the researcher selects the sample sizes for each group and specifies only $\mathrm{P}^{*}$, the probability of a correct selection. He then makes his selections by the following rule that is followed for each j: 
Assert that Group $j$ is better than the Control Group $C$ if $x_{j}$, the number of responses in Group $j$, satisfies

$$
x_{j} \geq x_{C}-d \sqrt{\bar{n}} / 2,
$$

where $x_{C}$ is the number of responses in the Control Group, $\bar{n}$ is the square-mean-root sample size, and $d$ is found from Table 3 as the entry for $k$, the total number of groups including the control, and the specified $\mathrm{P}^{*}$. (This, rule is an approximate solution based on the normal approximation to the binomial.)

Those groups selected by this rule are asserted to be better than the Control Group, and $\mathrm{P}^{*}$ is the overall confidence level for the joint statement that each group not included is worse than the Control Group in the sense that it has a significantly lower response rate. If no group is selected by this rule, we conclude that none is better than the Control.

This type of problem arose in Hansen (1980) where the experiment compared response rates for groups with a monetary incentive, a nonmonetary incentive of comparable value, and a control group of no incentive. The stated research hypothesis was that response rates are higher for both monetary and nonmonetary incentive groups than for the control group. The reported data are as follows: 
$\underline{\text { Responses }}$

Monetary

Nonmonetary

Control
308

177

114
Sample Size

810

804

811

A chi-square test was used on these data to confirm the hypothesis. The only appropriate statistical conclusion from this chi-square test is that the response rates are not all the same. On the other hand, our subset selection procedure is eminently appropriate to confirm the hypothesis with, say, 95\% confidence by the following methodology. Here we have $x_{C}=114, x_{1}=308, x_{2}=177, k=3$, and $\bar{n}=808$. Table 3 gives $d=2.71$ for $P^{*}=.95, k=3$, so that the selection rule is given by $x_{j} \geq 114-38.5=75.5$. The conclusion then is that both the Monetary and Nonmonetary incentive groups are better than the Control, and we have 95\% confidence that this assertion is correct.

A subset selection procedure, rather than a chi-square test, would have also been appropriate in other studies such as Walker and Burdick (1977), and Capon and Farley (1976).

\section{$\underline{\text { Discussion }}$}

In the last decade or so, a great number of articles in the statistical literature have been devoted to the various problems of selection procedures. These procedures should be of considerable interest to marketing researchers, as they provide a precise and easy to apply methodology for the solution of many research problems. 
The purpose of this paper was to introduce the readers of this journal to the methodology of selection procedures. While only the essential ingredients of those procedures were covered in this paper, selection procedures are now available which, unlike the traditional approaches such as chi-square analysis or analysis of variance, allow the treatment of most selection problems directly as a selection problem with appropriate evaluation of the probability of being correct. In addition to the examples presented here these procedures can be applied to a variety of frequently encountered research problems, such as the selection of the population with the largest multiple correlation coefficient, the selection of the normal population with the best regression value or the selection of the normal population with the smallest variance. Moreover, as mentioned earlier, the essential ta'oles required for carrying out these procedures are now available in usable form in Gibbons, Olkins, and Sobel (1977) for most common distributions such as the normal, binomial, multinomial and so on, as well as for some nonparametric problems. To the authors' knowledge, applications of these kinds of statistical procedures have not been published in marketing related studies with the exception of Dalal and Srinavasan (1977) who applied the indifference zone approach to determine the sample size required to pre-test the comparative effectiveness of advertising copies. The methods have been applied in several other: areas, however. Applications in agricultural 
and animal science include Becker (1961, 1962, 1964), Chew (1977), and Soller and Putter (1964, 1965). Management science applications in the literature include Kleijnen (1975), Kleijnen, Naylor, and Seaks (1972), and Schmidt and Taylor (1970). Applications to selecting the best economic policy are in Naylor (1971), and Naylor, Wertz, and Wonnacott (1968). Naylor, Wertz, and Wonnacott (1967) used the methods to analyze data from computer simulations, and Mamrak and Amer (1979) used the techniques to select among interactive computer services. 
Table 1

Smallest Sample Size $n$ Needed to Satisfy the $\left(\delta^{*}, \mathrm{P}^{*}\right)$ Requirement in Selecting the Binomial Population with the Largest Probability when $\mathrm{k}=3$

\begin{tabular}{|c|c|c|c|c|c|c|c|c|}
\hline$\delta^{*}$ & .50 & .60 & .75 & .80 & .85 & .90 & .95 & .99 \\
\hline .05 & 31 & 79 & 206 & 273 & 364 & 498 & 735 & 1308 \\
\hline .10 & 8 & 20 & 52 & 69 & 91 & 125 & 184 & 327 \\
\hline .15 & 4 & 9 & 23 & 31 & 41 & 55 & 82 & 145 \\
\hline .20 & 3 & 5 & 13 & 17 & 23 & 31 & 46 & 81 \\
\hline .25 & 2 & 4 & 9 & 11 & 15 & 20 & 29 & 52 \\
\hline .30 & 2 & 3 & 6 & 8 & 10 & 14 & 20 & 35 \\
\hline .35 & 2 & 2 & 5 & 6 & 8 & 10 & 15 & 26 \\
\hline .40 & 1 & 2 & 4 & 5 & 6 & 8 & 11 & 20 \\
\hline .45 & 1 & 2 & 3 & 4 & 5 & 6 & 9 & 15 \\
\hline .50 & 1 & 2 & 3 & 3 & 4 & 5 & 7 & 12 \\
\hline
\end{tabular}


Table 2

Smallest Sample Size $n$ Needed to Satisfy the $\left(\delta^{*}, \mathrm{P}^{*}\right)$ Requirement in Selecting the Binomial Population with the Largest Probability when $\mathrm{k}=4$

\begin{tabular}{|c|c|c|c|c|c|c|c|c|}
\hline$\delta^{*}$ & .50 & .60 & .75 & .80 & .85 & .90 & .95 & 99 \\
\hline .05 & 71 & 134 & 283 & 359 & 458 & 601 & 850 & 1442 \\
\hline .10 & 18 & 34 & 71 & 90 & 114 & 150 & 212 & 360 \\
\hline .15 & 8 & 15 & 32 & 40 & 51 & 67 & 94 & 160 \\
\hline .20 & 5 & 9 & 18 & 23 & 29 & 38 & 53 & 89 \\
\hline .25 & 3 & 6 & 12 & 14 & 18 & 24 & 34 & 57 \\
\hline .30 & 3 & 4 & 8 & 10 & 13 & 17 & 23 & 39 \\
\hline .35 & 2 & 3 & 6 & 7 & 9 & 12 & 17 & 28 \\
\hline .40 & 2 & 3 & 5 & 6 & 7 & 9 & 13 & 21 \\
\hline .45 & 2 & 2 & 4 & 5 & 6 & 7 & 10 & 17 \\
\hline .50 & 2 & 2 & 3 & 4 & 5 & 6 & 8 & 13 \\
\hline
\end{tabular}


Table 3

Values of $\tau$ for Fixed $\mathrm{P}$ (or $\mathrm{P}^{*}$ )

\begin{tabular}{c|cccccc}
\hline & \multicolumn{6}{|c}{$P\left(\right.$ or $\left.P^{*}\right)$} \\
$k$ & .750 & .900 & .950 & .975 & .990 & .999 \\
\hline 2 & 0.9539 & 1.8124 & 2.3262 & 2.7718 & 3.2900 & 4.3702 \\
3 & 1.4338 & 2.2302 & 2.7101 & 3.1284 & .3 .6173 & 4.6450 \\
4 & 1.6822 & 2.4516 & 2.9162 & 3.2220 & 3.7970 & 4.7987 \\
5 & 1.8463 & 2.5997 & 3.0552 & 3.4532 & 3.9196 & 4.9048 \\
& & & & & & \\
6 & 1.9674 & 2.7100 & 3.1591 & 3.5517 & 4.0121 & 4.9855 \\
7 & 2.0626 & 2.7972 & 3.2417 & 3.6303 & 4.0860 & 5.0504 \\
8 & 2.1407 & 2.8691 & 3.3099 & 3.6953 & 4.1475 & 5.1046 \\
9 & 2.2067 & 2.9301 & 3.3679 & 3.7507 & 4.1999 & 5.1511 \\
& & & & & & \\
10 & 2.2637 & 2.9829 & 3.4182 & 3.7989 & 4.2456 & 5.1916 \\
15 & 2.4678 & 3.1734 & 3.6004 & 3.9738 & 4.4121 & 5.3407 \\
20 & 2.6009 & 3.2986 & 3.7207 & 4.0899 & 4.5230 & 5.4409 \\
25 & 2.6987 & 3.3911 & 3.8099 & 4.1761 & 4.6057 & 5.5161 \\
\hline
\end{tabular}




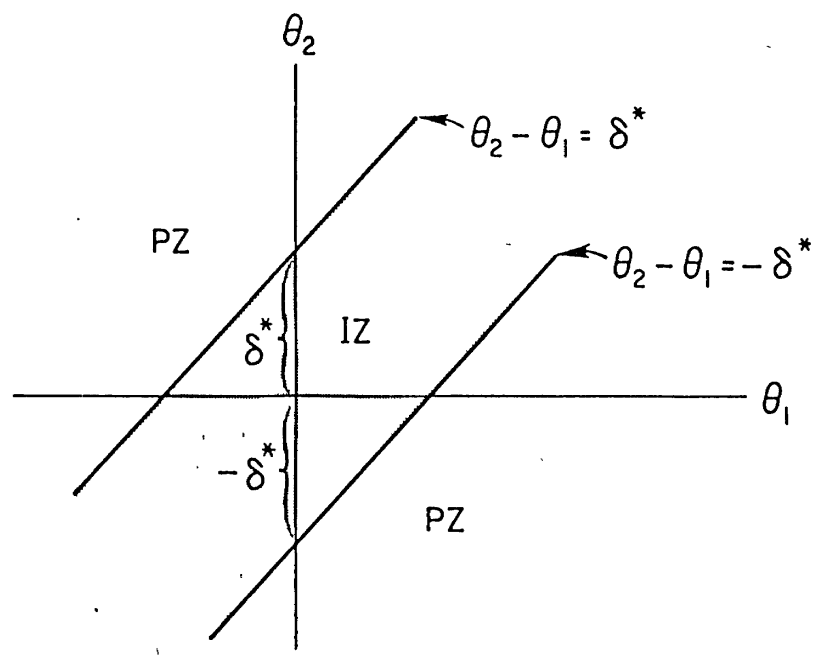

Figure 1. Preference Zore is $\left|\theta_{2}-\theta_{1}\right| \geq \delta *$ 


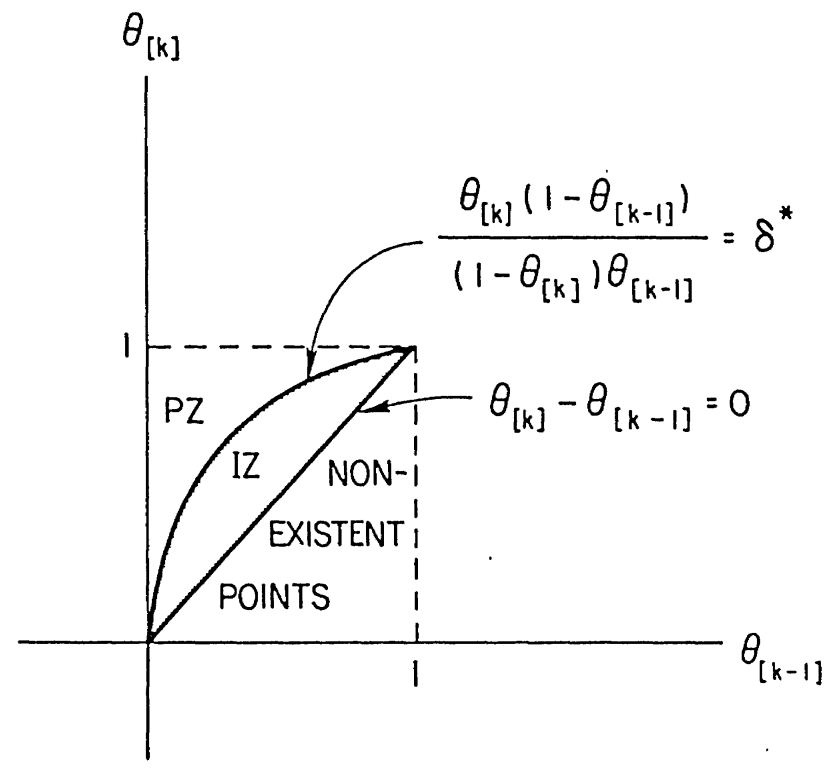

Figure 2. Preference Zone is $\theta_{[k]}\left(1-\theta_{[k-1]}\right) /\left(1-\theta_{[k]}\right) \theta_{[k-1]} \geq \delta *$. 


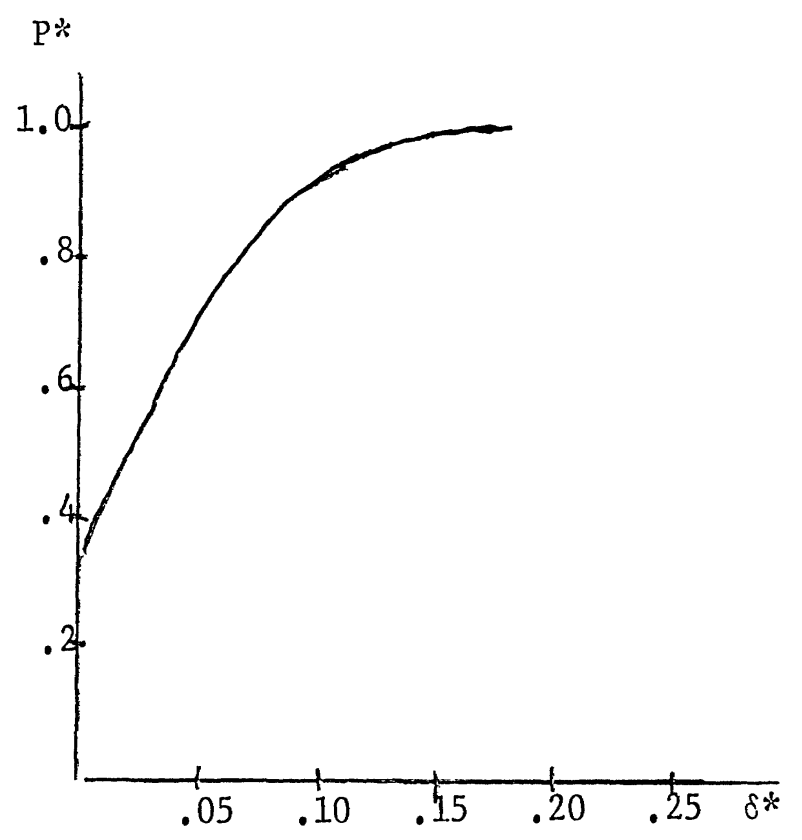

Figure 3. Operating Characteristic Curve for the Froblem of Selecting the One Binomial Population with the Largest Probabijity of Success when $k=3, n=1.50$ 
REFERENCES

Bechhofer, R. E. (1954), "A Single-Sample Multiple Decision Procedure for Ranking Means of Normal Populations with Known Variances," Annals of Mathematical Statistics, 25, (March), 16-39.

, C. W. Dunnett, and M. Sobel (1954),

"A Two-Sample Multiple Decision Procedure for Ranking

Means of Normal Populations with a Common Unknown

Variance," Biometrika, 41, (June), 170-176.

Becker, W. A. (1961), "Comparing Entries in Random Sample Tests," Poultry Science, 40, (November), 1507-14. , (1962), "Ranking All-Or-None Traits in Random

Sample Tests," Poultry Science, 41, (September), 1437-8. , (1964), "Changes in Performance of Entries in

Random Sample Tests," Poultry Science, 43, (May), 716-22. Capon, Noel and John U. Farley (1975), "The Impact of Message on Direct Mail Responses," Journal of Advertising Research, 16, (October), 69-75. Chew, Victor (1977), Comparisons Among Treatment Means in an Analysis of Variance, Washington, D.C.: U.S. Government Printing Office, 228-258.

Dalal, S. R. and V. Srinivasan (1977), "Determining Sample Size for Pretesting Comparative Effectiveness of Advertising Copies," Management Science, 23, (August), 1284-94. Gibbons, Jean Dickinson, Ingram Olkin, and Milton Sobel (1977), Selecting and Ordering Populations: A New Statistical Methodology. New York: John Wiley \& Sons. 
Girshick, M. A. (1946), "Contributions to Sequential

Analysis: I," Annals of Mathematical Statistics, 17, (June), 123-143.

Goldstein, Larry and Hershey H. Friedman (1975), "A Case for

Double Postcards in Surveys," Journal of Advertising Research, 15, (April), 43-47.

Goodstadt, Michael S., Linda Chung, Reena Kronitz, and Gaynoll Cook (1977), "Mail Survey Response Rates: Their Manipulation and Impact," Journal of Marketing Research, 14, (August), 391-395. Goulet, Waldemar M. (1977,) "Efficacy of a Third Request Letter in Mail Surveys of Professionals," Journal of Marketing Research, 14, (February), 112-114.

Gupta, S. S. (1956), "On a Decision Rule for a Problem in Ranking Means," Unpublished Ph.D. thesis, Institute of Statistics, Mimeo Series No. 150, Chapel Hill: University of North Carolina.

Hansen, Robert A. (1980), "A Self-Perception Interpretation of the Effect of Monetary and Nonmonetary Incentives on Mail Survey Respondent Behavior," Journal of Marketing Research, 17, (February), 77-83.

Kleijnen, J. P. C. (1975), Statistical Techniques in Simulation,

Part II, New York: Marcel Dekker, Inc. , T. H. Naylor, and T. G. Seaks (1972),

"The Use of Multiple Ranking Procedures to Analyze

Simulations of Management Systems: A Tutorial," Management Science, Applications Series, 18, (Feburary), B245-57. 
Lipstein, Benjamin (1975), "In Defense of Small Samples," Journal of Advertising Research, 15, (February), 33-40. Mamrak, Sandra A., and P. D. Amer (1979), Computer Science \&

Technology: A Methodology for the Selection of

Interactive Computer Services, Washington, D. C.:

U.S. Government Printing Office, National Bureau of

Standards Special Publication 500-44.

Naylor, T. H. (1971), Computer Simulation Experiments with

Models of Economic Systems, New York: John Wiley \& Sons.

, K. Wertz and T. H. Wonnacott (1967), Methods

for Analyzing Data from Computer Simulations,"

Communications of the ACM, 10, (November), 703-10.

, K. Wertz and T. H. Wonnacott (1968), "Sorne

Methods for Evaluating the Effects of Economic Policies

Using Simulation Experiments," Review of the

International Statistical Institute, 36, 184-200.

Orpen, Christopher (1975), "Reaction to Black and White

Models," Journal of Advertising Research, 15, (October), $75-79$.

Robertson, Dan H. and Danny N. Bellenger (1978), "A New

Method of Increasing Mail Survey Responses: Contributions to Charity," Journal of Marketing Research, 15, (November), $623-633$.

Schmidt, J. W. and R. E. Taylor (1970), Simulation and Analysis of Industrial Systems, Richard Irwin, Homewood, Illinois. 
Shosteck, Herschel (1975), "Shopping, Voting and Media Preference," Journal of Advertising Research, 15, (December), 35-38.

Soller, M., and J. Putter (1964), "On the Probability that the Best Chicken Stock Will Come Out Best in a Single Random Sample Test," Poultry Science, 43, (November), $1425-27$.

(1965), "Probability of Correct Selection of

Sires Having Highest Transmitting Ability," Journal of Dairy Science, 48, (June), 747-48.

Wald, A. (1947), Sequential Analysis, New York: John Wiley \& Sons.

Walker, Bruce J. and Richard K. Burdick (1977), "Advance Correspondence and Error in Mail Surveys," Journal of - Marketing Research, 14, (August), 379-382. 